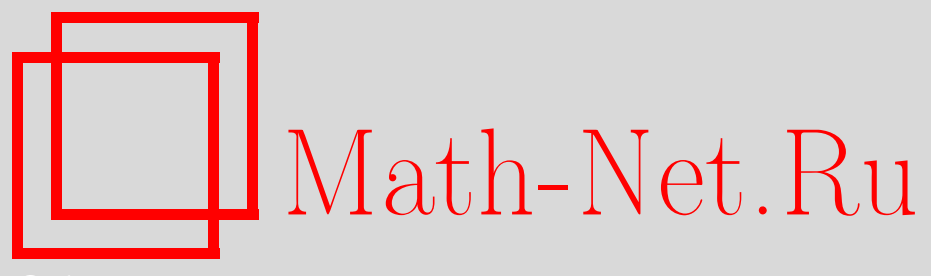

Ю. Л. Притыкин, Конечно-автоматные преобразования строго почти периодических последовательностей, $M a$ тем. заметки, 2006, том 80, выпуск 5, 751-756

DOI: https://doi.org/10.4213/mzm3084

Использование Общероссийского математического портала Math-Net.Ru подразумевает, что вы прочитали и согласны с пользовательским соглашением http://www . mathnet.ru/rus/agreement

Параметры загрузки:

IP : 54.224 .135 .184

26 апреля 2023 г., $17: 10: 27$

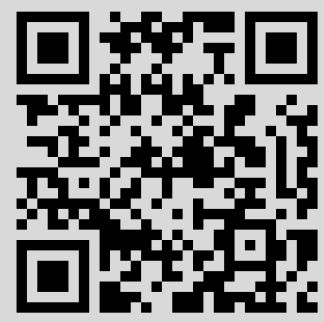




\section{КОНЕЧНО-АВТОМАТНЫЕ ПРЕОБРАЗОВАНИЯ СТРОГО ПОЧТИ ПЕРИОДИЧЕСКИХ ПОСЛЕДОВАТЕЛЬНОСТЕЙ}

\section{Ю. Л. Притыкин}

Различные варианты понятия почти периодичности являются естественным обобщением понятия периодичности. Понятие строгой почти периодичности возникло в символической динамике, но впоследствии также оказалось продуктивным в математической логике и теории алгоритмов. В работе рассмотрен класс существенно почти периодических последовательностей (т.е. строго почти периодических с приписанным в начале произвольным префиксом). Доказано, что свойство существенной почти периодичности сохраняется при конечно-автоматных преобразованиях, а также под действием конечных преобразователей. Класс существенно почти периодических последовательностей содержится в классе почти периодических последовательностей. Доказано, что это включение строгое.

Библиография: 4 названия.

1. Введение. Строго почти периодические последовательности (под другим названием) изучались в работах Морса и Хедлунда [1], [2], а также рядом других авторов. Это понятие возникло в символической динамике, но впоследствии оказалось продуктивным как в математической логике, так и в теории алгоритмов.

Класс автоматных образов строго почти периодических последовательностей (определения см. ниже), очевидно, содержит класс существенно почти периодических последовательностей, под которыми мы понимаем строго почти периодические с приписанным в начале произвольным префиксом. Действительно, для получения последовательности $a \omega$ из строго почти периодической последовательности $\omega$ можно воспользоваться так называемым конечным автоматом с задержкой: в его памяти хранится конечное слово $a$, и во время работы он сначала выдает это слово, а потом входную последовательность с задержкой $|a|$ (для этого автомат хранит в памяти последние $|a|$ символов последовательности). Основной результат статьи (теорема 2) заключается в том, что указанные классы совпадают. Другими словами, теорема 2 утверждает, что свойство существенной почти периодичности сохраняется при конечно-автоматных преобразованиях. В последней части статьи рассмотрено обобщение понятия конечного автомата - конечный преобразователь, и для него доказано аналогичное утверждение.

Работа выполнена при поддержке Российского фонда фундаментальных исследований, грант № 06-01-00122, и программы “Ведущие научные школы”, грант № 358.2003.1.

(C) Ю. Л. Притыкин, 2006 
Стоит заметить, что в [3] было рассмотрено расширение класса строго почти периодических последовательностей - класс почти периодических последовательностей (определение см. ниже). В частности, было доказано, что этот класс также замкнут относительно автоматных преобразований. Ясно, что класс почти периодических последовательностей содержит класс существенно строго почти периодических. Оказывается, это включение строгое (теорема 1).

Пусть $A$ - конечный алфавит. Будем рассматривать последовательности над этим алфавитом - отображения $\omega: \mathbb{N} \rightarrow A$ (где $\mathbb{N}=\{0,1,2, \ldots\}$ ). Через $A^{*}$ обозначим множество всех слов над алфавитом $A$. Если $i \leqslant j$ натуральные, то через $[i, j]$ обозначим отрезок натурального ряда с концами в $i$ и $j$, т.е. множество $\{i, i+1, i+2, \ldots, j\}$. Через $\omega[i, j]$ обозначим отрезок последовательности $\omega-$ слово $\omega(i) \omega(i+1) \ldots \omega(j)$. Говорят, что $[i, j]-$ вхождение в последовательность $\omega$ слова $x \in A^{*}$, если $\omega[i, j]=x$. Через $|x|$ будем обозначать длину слова $x$. Мы представляем себе последовательность расположенной горизонтально и идущей слева направо до бесконечности, поэтому будем использовать выражения “левее" и "правее" для меньших и бо́льших индексов соответственно.

2. Почти периодичность. Последовательность $\omega$ называется почти периодической, если для каждого слова $x$, входящего в нее бесконечное число раз, найдется такое натуральное $l$, что на каждом отрезке длины $l$ последовательности $\omega$ найдется вхождение слова $x$.

Последовательность $\omega$ называется строго почти периодической, если для каждого слова $x$, входящего в нее хотя бы один раз, найдется такое натуральное $l$, что на каждом отрезке длины $l$ последовательности $\omega$ найдется вхождение слова $x$.

Для удобства введем еще одно определение. Будем называть последовательность $\omega$ существенно строго почти периодической, если она есть конкатенация некоторого конечного слова и строго почти периодической последовательности.

Каждая существенно строго почти периодическая последовательность, очевидно, является почти периодической. Покажем, что класс почти периодических последовательностей строго шире класса существенно строго почти периодических.

Теорема 1. Существует почти периодическая последовательность над алфавитом $\{0,1\}$, не являющаяся существенно строго почти периодической.

ДокАЗАтЕЛЬСтво. Построим цепочку двоичных слов: $a_{0}=1, a_{1}=10011, a_{2}=$ 1001101100011001001110011 и т.д. Слово $a_{n+1}$ получается из $a_{n}$ по такому правилу:

$$
a_{n+1}=a_{n} \bar{a}_{n} \bar{a}_{n} a_{n} a_{n}
$$

где $\bar{x}$ обозначает слово, полученное из слова $x$ заменой всех символов 1 на 0 и 0 на 1. Введем обозначение

$$
c_{n}=\underbrace{a_{n} a_{n} \ldots a_{n}}_{10}
$$

и рассмотрим последовательность

$$
\omega=c_{0} c_{1} c_{2} c_{3} \ldots
$$

Докажем, что она и является искомой. 
Длина $a_{n}$ равна $5^{n}$, поэтому длина начального отрезка $c_{0} c_{1} \ldots c_{n-1}$ последовательности $\omega$ равна

$$
10\left(1+5+\cdots+5^{n-1}\right)=\frac{5}{2}\left(5^{n}-1\right) .
$$

Для удобства введем обозначение

$$
l_{n}=\frac{5}{2}\left(5^{n}-1\right)=\left|c_{0} c_{1} \ldots c_{n-1}\right|
$$

Покажем, что $\omega$ почти периодична. Пусть непустое слово $x$ встречается в $\omega$ бесконечное количество раз. Возьмем такое $n$, что $|x|<5^{n}$. Пусть $[i, j]-$ вхождение слова $x$ в $\omega$ такое, что $i \geqslant l_{n}$. Из построения последовательности следует, что для каждого $k$ всю последовательность $\omega$, начиная с позиции $l_{k}$, можно рассматривать не только как конкатенацию символов 0 и 1 , но и как конкатенацию слов $a_{k}$ и $\bar{a}_{k}$. Поэтому в силу выбора $i$ слово $x$ является подсловом одного из четырех слов $a_{n} a_{n}$, $a_{n} \bar{a}_{n}, \bar{a}_{n} a_{n}, \bar{a}_{n} \bar{a}_{n}$. Заметим, что слово 10011 содержит всевозможные слова длины два $00,01,10,11$, поэтому слово $a_{n+1}$ содержит каждое из слов $a_{n} a_{n}, a_{n} \bar{a}_{n}, \bar{a}_{n} a_{n}$, $\bar{a}_{n} \bar{a}_{n}$. Отсюда получаем, что слово $x$ является подсловом слова $a_{n+1}$. Аналогично, $x$ является подсловом слова $\bar{a}_{n+1}(01100$ тоже содержит каждое из слов $00,01,10,11)$. На каждом отрезке длины $2\left|a_{n+1}\right|$, расположенном правее позиции $l_{n+1}$, найдется вхождение слова $a_{n+1}$ или $\bar{a}_{n+1}$, поэтому для $l=\frac{5}{2}\left(5^{n+1}-1\right)+2 \cdot 5^{n+1}$ выполнено, что на каждом отрезке длины $l$ найдется вхождение слова $x$.

Докажем теперь для каждого натурального $n>0$, что слово $c_{n}$ не встречается в последовательности $\omega$ правее позиции $l_{n+1}$. Тем самым мы покажем, что для последовательности, полученной из $\omega$ отрезанием начального отрезка длины не более $l_{n}$, найдется слово, а именно, $c_{n}$, которое входит в нее ненулевое конечное количество раз, т.е. такая последовательность не является строго почти периодической. Таким образом, будет доказано, что $\omega$ не является существенно строго почти периодической.

Пусть $\nu$ - последовательность, полученная из $\omega$ отрезанием начального отрезка длины $l_{n+1}$. Как уже было замечено, для каждого $k, 1 \leqslant k \leqslant n+1$, ее можно представить как конкатенацию слов $a_{k}$ и $\bar{a}_{k}$. Предположим, $c_{n}$ входит в $\nu$, и пусть $[i, j]$ - одно из таких вхождений. При $n>0$ слово $c_{n}$ начинается с $a_{1}$, поэтому $[i, i+4]$ является вхождением слова $a_{1}$ в $\nu$. Заметим, что если $a_{1}=10011$ входит в $a_{1} a_{1}=1001110011, a_{1} \bar{a}_{1}=1001101100, \bar{a}_{1} a_{1}=0110010011$ или $\bar{a}_{1} \bar{a}_{1}=0110001100$, то только с нулевой или с пятой позиции. Поэтому $5 \mid i$, т.е. начало вхождения слова $c_{n}$ в $\nu$ совпадает с началом какого-нибудь из слов $a_{1}$ и $\bar{a}_{1}$, из которых, можно считать, и составлено $\nu$. Индукцией по $m$ докажем, что $5^{m} \mid i$ при $1 \leqslant m \leqslant n$, т.е. если представить $\nu$ в виде конкатенации слов $a_{m}$ и $\bar{a}_{m}$, то начало вхождения $c_{n}$ совпадает с началом одного из этих слов. База для $m=1$ уже доказана. Зная это утверждение для $m=k$, мы можем представить $\nu$ и $c_{n}$ составленными из "букв" $a_{k}$ и $\bar{a}_{k}$, причем $c_{n}$ входит в $\nu$. Тогда, чтобы доказать утверждение для $m=k+1$, можно применить точно такое же рассуждение, как и в случае $m=1$, только заменить 1 и 0 на $a_{k}$ и $\bar{a}_{k}$, и $a_{1}$ и $\bar{a}_{1}$ на $a_{k+1}$ и $\bar{a}_{k+1}$, и при этом использовать, что $c_{n}$ начинается c $a_{k+1}$. 
Таким образом, мы показали, что $5^{n} \mid i$, т.е. если представлять себе $\nu$ и $c_{n}$ составленными из "букв" $a_{n}$ и $\bar{a}_{n}$, то мы доказали, что

$$
c_{n}=\underbrace{a_{n} a_{n} \ldots a_{n}}_{10}
$$

входит в $\nu$. Но на каждом отрезке последовательности $\nu$ из 10 подряд идущих "букв" $a_{n}$ и $\bar{a}_{n}$ можно найти вхождение "пятибуквенного" слова $a_{n+1}$ или $\bar{a}_{n+1}$, а в нем вхождение "буквы" $\bar{a}_{n}$. Противоречие.

Более того, из доказательства видно, как построить бесконечное, даже континуальное множество почти периодических последовательностей, не являющихся существенно строго почти периодическими. Можно действовать, например, так: каждой последовательности $\tau: \mathbb{N} \rightarrow\{9,10\}$ сопоставить последовательность, построенную так же, как и $\omega$ из доказательства теоремы 1 , только в качестве $c_{n}$ брать слово

$$
c_{n}^{(\tau)}=\underbrace{a_{n} a_{n} \ldots a_{n}}_{\tau(n)} .
$$

Ясно, что построенные таким образом $\omega_{\tau}$ будут различны для различных $\tau$ (для различных $\tau_{1}$ и $\tau_{2}$ достаточно рассмотреть минимальное $n$, в котором они различаются: тогда в $c_{n}^{\left(\tau_{1}\right)}$ и $c_{n}^{\left(\tau_{2}\right)}$ разное количество $a_{n}$, но после них в $\omega_{\tau_{1}}$ и $\omega_{\tau_{2}}$ идет $\left.a_{n+1}=a_{n} \bar{a}_{n} \bar{a}_{n} a_{n} a_{n}\right)$. Осталось заметить, что всевозможных $\tau$ континуум.

3. Автоматные преобразования. Конечным автоматом назовем совокупность $F=\langle A, B, Q, \widetilde{q}, f\rangle$, где $A$ и $B$ - конечные множества, называемые соответственно входной и выходной алфавит, $Q$ - конечное множество состояний, $\widetilde{q} \in Q-$ выделенное состояние, называемое начальным, и

$$
f: Q \times A \rightarrow Q \times B
$$

- функция переходов. Последовательность $\left\langle p_{n}, \beta(n)\right\rangle_{n=0}^{\infty}$, где $p_{n} \in Q, \beta(n) \in B$ назовем автоматным преобразованием последовательности $\alpha$ из букв алфавита $A$, если $p_{0}=\widetilde{q}$ и для каждого $n$ выполняется $\left\langle p_{n+1}, \beta(n)\right\rangle=f\left(p_{n}, \alpha(n)\right)$. Последовательность $\beta$ назовем тогда результатом преобразования $\alpha$ автоматом $F$ и обозначим $F(\alpha)$. Очевидно, для каждого $F$ и $\alpha$ результат $F(\alpha)$ существует и определен однозначно. Если $[i, j]$ - вхождение слова $x$ в последовательность $\alpha$, причем $p_{i}=q$, то будем говорить, что автомат $F$ подходит к этому вхождению слова $x$ в состоянии $q$.

В [3] было доказано следующее утверждение: если $F$ - конечный автомат, $\omega$ почти периодическая последовательность, то $F(\omega)$ тоже почти периодическая.

Оказывается, эту теорему можно дополнить.

Теорема 2. Если F-конечный автомат, $\omega$ - существенно почти периодическая последовательность, то $F(\omega)$ тоже существенно строго почти периодическая.

ДокАзАтЕЛЬСтво. Очевидно, что достаточно доказать теорему для $\omega$ - строго почти периодических, так как существенно строго почти периодическая последовательность (в нашем случае $F(\omega)$ ) остается существенно строго почти периодической при приписывании к ней произвольного префикса. 
Итак, пусть $\omega$ - строго почти периодическая последовательность. По указанной выше теореме последовательность $F(\omega)$ почти периодическая. Предположим, что она не является существенно строго почти периодической. Это означает, что для любого натурального $N$ можно найти такое слово, которое входит в $F(\omega)$ правее позиции $N$, и после этого уже не входит. Действительно, отрезав от $F(\omega)$ начальный отрезок $[0, N]$, мы не получим строго почти периодическую последовательность, значит, найдется слово, входящее в нее ненулевое конечное количество раз. Тогда возьмем самое правое вхождение этого слова.

Пусть $\left[i_{0}, j_{0}\right]$ - самое правое вхождение некоторого слова $y_{0}$ в последовательность $F(\omega)$. Для некоторого натурального $l_{0}$ слово $x_{0}=\omega\left[i_{0}, j_{0}\right]$ входит в любой отрезок длины $l_{0}$ последовательности $\omega$ (в силу строгой почти периодичности). При этом если $q_{0}$ - состояние, в котором автомат $F$ подошел к позиции $i_{0}$, то ко всем дальнейшим вхождениям слова $x_{0}$ в $\omega$ автомат не может подойти в состоянии $q_{0}$, иначе бы он выдал полностью слово $y_{0}$.

Пусть теперь $[r, s]$ - самое правое вхождение некоторого слова $a$ в последовательность $F(\omega)$, причем $r>i_{0}+l_{0}$. На отрезке $\omega\left[r-l_{0}, r\right]$ найдется вхождение $\left[r^{\prime}, s^{\prime}\right]$ слова $x_{0}$, причем в силу выбора $r$ будет выполнено $r^{\prime}>i_{0}$. Положим тогда

$$
i_{1}=r^{\prime}, \quad j_{1}=s, \quad x_{1}=\omega\left[i_{1}, j_{1}\right], \quad y_{1}=F(\omega)\left[i_{1}, j_{1}\right] .
$$

Поскольку слово $а$ не входит в $F(\omega)$ правее позиции $r$, то и слово $y_{1}$, которое содержит $a$ в качестве подслова, не входит в $F(\omega)$ правее позиции $i_{1}$. Это значит, что если $q_{1}$ - состояние, в котором автомат подошел к позиции $i_{1}$, то больше никогда в состоянии $q_{1}$ к вхождению слова $x_{1}$ в последовательность $\omega$ автомат не подойдет. Поскольку $x_{1}$ начинается со слова $\omega\left[r^{\prime}, s^{\prime}\right]=x_{0}$, и $r^{\prime}>i_{0}$, то $q_{1} \neq q_{0}$. Таким образом, мы нашли слово $x_{1}$, ко всем вхождениям которого, расположенным правее $i_{1}$, автомат не может подойти, находясь в состояниях $q_{0}$ или $q_{1}$.

Пусть $m=|Q|$ - количество возможных состояний автомата. Продолжая так дальше рассуждать по индукции, мы построим цепочку слов $x_{k}=\omega\left[i_{k}, j_{k}\right]$ и соответствующих различных состояний $q_{k}$, где $k<m$, так что ко всем вхождениям $x_{k}$ в $\omega$ правее $i_{k}$ автомат не может подойти в состояниях $q_{0}, q_{1}, \ldots, q_{k}$. При $k=m$ получаем противоречие.

4. Конечные преобразователи. Пусть $A, B$ - конечные алфавиты. Отображение $h: A^{*} \rightarrow B^{*}$ называется гомоморфизмом, если для любых $u, v \in A^{*}$ выполнено $h(u v)=h(u) h(v)$. Ясно, что гомоморфизм полностью определяется своими значениями на однобуквенных словах. Если $\omega$ - последовательность букв алфавита $A$, по определению положим

$$
h(\omega)=h(\omega(0)) h(\omega(1)) h(\omega(2)) \ldots
$$

Пусть $h: A^{*} \rightarrow B^{*}$ - гомоморфизм, $\omega$ - почти периодическая последовательность над алфавитом $A$. В [3] было показано, что последовательность $h(\omega)$ будет снова почти периодической. Ясно тогда, что для $\omega$ строго почти периодической $h(\omega)$ будет строго почти периодической. Действительно, достаточно показать, что для любого слова $u=\omega[i, j]$ слово $h(u)=h(\omega(i)) \ldots h(\omega(j))$ встречается в $h(\omega)$ бесконечно много раз. Но это следует из определения $h(\omega)$ и из того, что $\omega$ строго почти периодична, и значит, слово $u$ встречается в ней бесконечно много раз. Очевидно, 
для $\omega$ существенно почти периодической $h(\omega)$ будет снова существенно строго почти периодической.

Естественным обобщением понятия конечного автомата является понятие конечного преобразователя (подробнее см. [3], [4]). Отличие состоит в том, что конечный преобразователь может выдавать слово произвольной длины, прочитав один символ входа. Формально, меняется только определение функции переходов: теперь она имеет вид $f: Q \times A \rightarrow Q \times B^{*}$. Если последовательность $\left\langle p_{n}, v_{n}\right\rangle_{n=0}^{\infty}$, где $p_{n} \in Q$, $v_{n} \in B^{*}$, является преобразованием последовательности $\alpha$, то результатом преобразования назовем последовательность $v_{0} v_{1} v_{2} \ldots$.

Действие конечного преобразователя можно представить в виде композиции автоматного преобразования и гомоморфизма. Каждый из этих типов преобразований, как мы знаем, сохраняет свойство почти периодичности, поэтому получаем следствие: почти периодические последовательности под действием конечных преобразователей переходят в почти периодические. Аналогично, из теоремы 2 и сохранения существенной строгой почти периодичности при гомоморфных отображениях следует, что конечные преобразователи сохраняют также и свойство существенной почти периодичности.

Автор выражает искреннюю благодарность А.Л. Семенову и А. А. Мучнику за привлечение внимания автора к этой теме, а также за ценные и полезные замечания и советы по поводу настоящей работы.

\section{СПИСОК ЦИТИРОВАННОЙ ЛИТЕРАТУРЫ}

[1] M. Morse, G. A. Hedlund, "Symbolic dynamics", Amer. J. Math., 60 (1938), 815-866.

[2] M. Morse, G. A. Hedlund, "Symbolic dynamics II: Sturmian trajectories", Amer. J. Math., 62 (1940), 1-42.

[3] An. Muchnik, A. Semenov, M. Ushakov, "Almost periodic sequences", Theoret. Comput. Sci., 304 (2003), 1-33.

[4] A. Weber, "On the valuedness of finite transducers", Acta Informatica, 27 (1989), 749-780.

Ю. Л. Притыкин

Московский государственный университет

им. М. В. Ломоносова

E-mail: pritykin@lpcs.math.msu.su
Поступило

27.06.2005 